\title{
Necessity of Educational Reconsideration with Reference to Shielding Responsibilities
}

\author{
Saeed Shoja Shafti* \\ University of Social Welfare and Rehabilitation Sciences (USWR)Razi Psychiatric Hospital Tehran, Iran \\ *Corresponding author: Saeed Shoja Shafti, Full Professor of Psychiatry, University of Social Welfare and Rehabilitation Sciences \\ (USWR)Razi Psychiatric Hospital Tehran, Iran
}

\begin{abstract}
Recent pandemic of coronavirus has exposed that Homo sapiens is still very vulnerable against the microorganisms, which has been ascertained in the past over and over again with respect to different biological or ecological threats. Modern technology and advancements, while very effective and helpful, could only decline the morbidly and mortality due to various communicable or non-communicable causes, not eradicating it. Hence, fighting against existing biotic or natural threats demands an all-inclusive and all-purpose group effort and mutual aid, if it is supposed to be effective. In the present pandemic, compliance of physicians and medical staff regarding recommended protective maneuvers, like making use of mask or gloves, or obeying the recommended interpersonal distance, has the leading role, in this regard. Without such considerations, practically, not merely in theory, challenging with national or universal dangers would not be realistic or achievable. But, while non-compliance of common people due to lack of scientific knowledge or unavailability of protective equipment can be understandable, non-compliance by some medical doctors and personnel, who have related information, cognizance and accessibility to needed objects, is absolutely improper and incomprehensible.
\end{abstract}

\section{Introduction}

Though, maybe, neither of existent protecting procedures nor the suggested guidelines may guarantee a complete safety against the biotic threats, surely exercising safety measures will heighten, firstly, the personnel's protection, and, then, the publics' health. But, why some of medical workers, in spite of earlier training and accessibility to related data and tools, avoid shielding exercises unless it is mandatory? In other words, what is wrong with the professional insight that is expected to be revealed by the trained workforces, especially if they have a symbolic duty, as well, to be an apt model for general public? How the morbid cycle of spreading of communicable diseases can be obstructed or weakened when the most important warriors of battle do not have faith in their armaments, even figuratively, up to development of healthier approaches. As is known, professional insight depends on theoretic knowledge and practical training. Theoretic knowledge cannot be creative if it is not accompanied with thorough intellectual capacity and comprehension of core concept. Practical training, as well, cannot be fruitful if it is not supplemented with through formulation and rehearsal of grasped data in real settings. These two processes form the core curriculum of every academe and department with respect to pedagogic preparations. If educations about biotic dangers and associated precautionary stratagems are among the basic lessons of physicians and clinical staffs, then why resistance is still evident in some GPs, specialists and workforces concerning compatibility with defensive guidelines and policies. Unfortunately, such a noncompliance makes them as like as untutored populace with respect to possibility of transmission of contagious diseases and perpetuation of a sequence that demands group struggle if it is going to be controlled or exterminated. Disregard to plausible psychological factors, like passive-aggressive, antisocial, histrionic, paranoid or schizotypal personality traits, or unconscious acting outs, like unconscious suicide attempt, or presence of depressive symptoms in a covered or exposed episode, like hopelessness, helplessness, lack of interest, which may intrude sensible judgment of every individual, this problem shows that logical or scientific insight is still governable by cultural ethics and environmental standards; Namely, cultural ideals can undermine professional or scientific insight if there is some kind of conflict between one's 
believes and existent facts. There are many cults and followers who have faith in strange healers, like witchdoctors, shamans, faith healers, and naturopaths, which seem omnipotent and free from faults or boundaries of conventional doctors and medications.

While usually a mixture of well-educated and ill-informed enthusiasts may make the admirers of such cults, hope to indefinite supremacy is a historical and universal inclination that may impact everybody, whether medical employees or non-medical workers. It creates optimism, which is priceless, matchless and irreplaceable. But, noncompliance to protective measures may speed up distribution of communicable illnesses, too. When it is being done by a health worker who has the least possible amount of information concerning the topic, but based on favored subjective faiths disregard the public safety, it is not just neglect or bias; it is similar to assassination, whether unintentional or deliberate. The situation is as like as military or traffic rules and recommendations that everybody should obey even if he or she dislikes them, and should reimburse if cause fatalities due to breaking the rules or lack of knowledge. Social rules have been thought up on behalf of shared life. Conjoint existence, as well, cannot sustain without

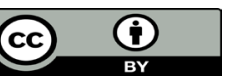

This work is licensed under Creative

Commons Attribution 4.0 License

To Submit Your Article Click Here: Submit Article reciprocal respect and cooperative partnership. Careless behavior of a medical physician or staff, due to private considers, is not allowable, because subjectivity is limitless, while objectivity has clear boundaries and evidences. Replacing prejudice with fairness, as well, demands a through national curriculum that enlightens the apprentices thoughtfully and methodically.

\section{Conclusion}

The schooling should have prepared the trainee's thoughts for a restructure. Reforming mind must be ready enough to distinct cultural values from professional ethics, public customs from social rules, individual considers from certified requirements, subjective decisions from objective judgments, disorganized tactics from systematic approaches, personal welfares from community safeties, own ideas from collective objectives, rumors from facts, unscientific verdicts from scientific discoveries, and finally, emotive insight from logical insight. Achievement of said intentions requires methodical teachings, reasonable tutors and appropriate learners. Without such groundwork, biotic threats, as like as other environmental hazards, may have concealed collaborators, which can be more intimidating than the prime danger.

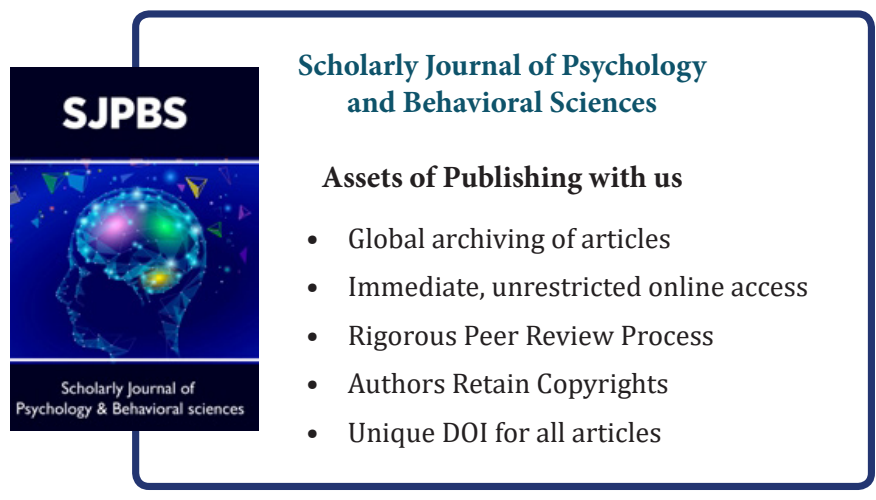

